\section{FRI0538 STRUCTURAL EFFECTS OF INTRA-ARTICULAR SPRIFERMIN IN SYMPTOMATIC RADIOGRAPHIC KNEE OSTEOARTHRITIS: A POST-HOC ANALYSIS OF CARTILAGE AND NON-CARTILAGINOUS TISSUE ALTERATIONS OF THE 2-YEAR DATA FROM A 5-YEAR RANDOMISED, PLACEBO-CONTROLLED, PHASE II STUDY}

F. Roemer ${ }^{1,2}$, J. Kraines ${ }^{3}$, A. Aydemir ${ }^{3}$, S. Wax 4 , M. Crema ${ }^{2}$, M. Hochberg ${ }^{5}$, A. Guermazi ${ }^{2} .{ }^{1}$ Radiology, University of Erlangen-Nuremberg, Erlangen, Germany; ${ }^{2}$ Radiology, Boston University School of Medicine, Boston; ${ }^{3} E M D$ Serono, Billerica; ${ }^{4}$ EMD Serono, Cambridge; ${ }^{5}$ Rheumatology, University of Maryland, Baltimore, USA

Background: Sprifermin, a recombinant human fibroblast growth factor 18 , is currently being investigated as a potential disease-modifying osteoarthritis (OA) drug. Recently, a dose-dependent increase in femorotibial cartilage thickness, as well as medial and lateral compartment cartilage, over two years was reported ${ }^{1}$. Objectives: The aim of this post-hoc analysis is to evaluate potential effects of sprifermin on additional structure endpoints, based on semi-quantitative MRI assessment over 24 months.

Methods: Patients aged 40-85 years with symptomatic radiographic knee OA, $\mathrm{KLG} 2$ or 3 , and medial $\mathrm{mJSW} \geq 2.5 \mathrm{~mm}$ in the target knee were randomised (1:1:1:1:1) to receive double-blinded placebo or sprifermin (30 $\mu \mathrm{g}$ or $100 \mu \mathrm{g})$, administered as 3 weekly intra-articular injections in cycles every 6 or 12 months. 1.5T or 3T MRIs were acquired at baseline, 6, 12, 18 and 24 month follow-up visits using a standard protocol (ClinicalTrials.gov identifier: NCT01033994).

MRIs were read using the Whole-Organ Magnetic Resonance Imaging Score (WORMS) system (time points of baseline, 12 and 24 months) by three trained musculoskeletal radiologists. Analyses of all sprifermin and placebo arms included multiple MRI-defined osteoarthritis features and multi-dimensional assessments: (a) delta-subregional approach (the difference in the number of subregions with worsening as compared to improvement) and (b) delta-sum approach (absolute scores of all subregions). Analyses were performed on a whole knee level and separately for medial, lateral, and patellofemoral compartments. To test for potential dose-response effects, Jonckheere-Terpstra (asymptotic) test was used. P-values were not adjusted for multiple testing.

Results: 549 patients were included. Dose-dependent treatment effect on cartilage morphology (i.e., less cartilage damage) was observed for the entire knee from baseline to 24 months using both delta sum and delta subregion approaches (table 1). For bone marrow lesions (BMLs), a dose-dependent treatment effect (improvement of BMLs) was observed from baseline to 24 months for the patellofemoral joint using both delta sum and delta subregion approaches but not the other compartments (table 2). No significant effects were seen for baseline to 24 month changes in Hoffa-synovitis, effusion-synovitis, menisci, or osteophytes.

Abstract FRI0538 - Table 1. Cartilage morphology change from baseline to 24 months, ITT analysis set

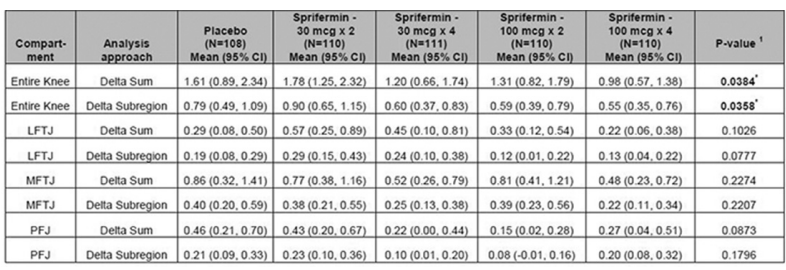

Abstract FRI0538 - Table 2. Bone marrow lesion change from baseline to 24 months, ITT analysis set

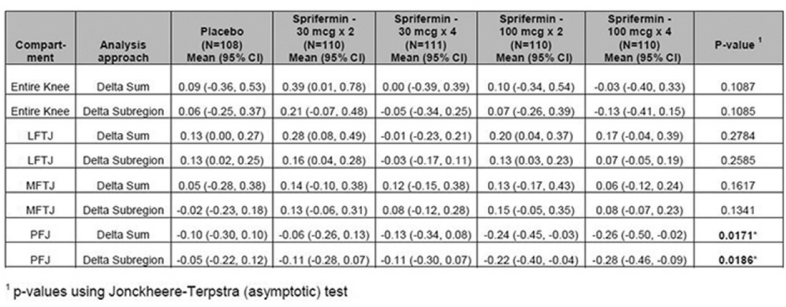

Conclusions: This post-hoc analysis indicates that sprifermin has a positive effect on cartilage morphology, in addition to the previously reported effect on cartilage thickness. Sprifermin was also associated with BML improvement in the patello-femoral joint. There were no significant effects associated with sprifermin on other joint tissues assessed, and no safety concerns raised.

\section{REFERENCE:}

[1] Hochberg MC, et al. Efficacy and Safety of Intra-Articular Sprifermin in Symptomatic Radiographic Knee Osteoarthritis: Results of the 2-Year Primary Analysis from a 5-Year Randomised, Placebo-Controlled, Phase II Study [abstract]. Arthritis Rheumatol 2017;69(suppl 10).

Disclosure of Interest: F. Roemer Shareholder of: Boston Imaging Core Lab (BICL), LLC., J. Kraines Employee of: EMD Serono, A. Aydemir Employee of EMD Serono, S. Wax Employee of: EMD Serono, M. Crema Shareholder of: Boston Imaging Core Lab (BICL), LLC., M. Hochberg Consultant for: Bioiberica SA Bristol Myers Squibb, EMD Serono, Galapagos, IBSA Biotechniq SA, Novartis Pharma AG, Pfizer Inc., Plexxikon, Samumed LLC, Theralogix LLC and TissueGene Inc, A. Guermazi Shareholder of: Boston Imaging Core Lab (BICL), LLC. Consultant for: to MerckSerono, TissueGene, GE, Pfizer, OrthoTrophix, AstraZeneca and Sanofi

DOI: 10.1136/annrheumdis-2018-eular.1960

\section{FRI0539 A SAFETY, TOLERABILITY, PHARMACOKINETICS (PK) AND PHARMACODYNAMICS (PD) STUDY WITH INCREASING ORAL DOSES OF GLPG1972 ADMINISTERED DAILY FOR 29 DAYS SHOWS A STRONG BIOMARKER EFFECT IN PATIENTS WITH KNEE AND/OR HIP OA}

H.M. Deckx ${ }^{1}$, S. Hatch ${ }^{1}$, M. Robberechts ${ }^{1}$, S. Dupont ${ }^{2}$, J. Desrivot ${ }^{3}$, H. Coleman ${ }^{4}$ S. Larsson ${ }^{5}$, A. Struglics ${ }^{5}$, E.M. van der Aar ${ }^{1}$, A. Fiew ${ }^{1} .{ }^{1}$ Clinical Development, Galapagos NV, Mechelen, Belgium; ${ }^{2}$ Translational Science; ${ }^{3}$ Galapagos SASU, Romainville, France; ${ }^{4} \mathrm{CRU}$, Covance, Daytona Beach, USA; ${ }^{5}$ Clinical Sciences, Lund University, Lund, Sweden

Background: Osteoarthritis $(\mathrm{OA})$ is characterised by structural changes of the joint, of which degradation of articular cartilage is one of the major signs ${ }^{1}$. The main proteoglycan component of the extracellular matrix of articular cartilage is aggrecan. GLPG1972 as a potent and selective inhibitor of ADAMTS-5, a key aggrecan-cleaving enzyme involved in cartilage degradation, is being developed as a potential disease-modifying OA drug (DMOAD). Aggrecan cleavage by ADAMTS- 5 results in release of N-terminal ARGS neo-epitope fragments of which serum levels significantly decreased in healthy subjects treated with GLPG1972 during 14 days in a previous study².

Objectives: To assess safety, tolerability, PK and PD (serum ARGS-aggrecan levels) during and following administration of GLPG1972 in patients with knee and/or hip OA.

Methods: This was a single centre, randomised, double-blind, placebo-controlled, age and gender stratified, ascending dose Phase lb study, with three semi-sequential cohorts of 10 patients each, randomised to active drug or placebo in a 4:1 ratio. Doses tested were once daily 100, 200 and $300 \mathrm{mg}$. Treatment duration was 29 days. Patients had follow-up visits 14 and 21 days after last dosing for additional PD assessments. Methods for PD have been described previously ${ }^{3}$.

Results: Thirty patients were included. Of these, 24 patients (M/F rate 8/16, 14 aged 50-64 and 10 aged 65-75) received active medication.

All adverse events (AE) were mild and transient. No serious AEs were reported during the study; one female patient in the $300 \mathrm{mg}$ group was discontinued after 15 days of treatment due to drug-related elevated transaminase values which returned to normal 9 days later while her bilirubin levels remained normal. There were no overall trends in lab abnormalities over time or significant changes in vita signs, ECG and Holter parameters.

Steady state in plasma exposure was reached after 3 days of dosing. Exposure increased dose-proportionally. Mean serum ARGS levels (SEM) decreased steadily over time in all patients receiving GLPG1972: $-40 \%(2.9), \%-46 \%(4.5)$ and $-53 \%$ (2.8) at day 15 compared to baseline in the 100,200 and $300 \mathrm{mg}$ group respectively. These levels remained stable until last dose on day 29 , then consis tently returned to pre-dose levels for all groups 14 and 21 days after last dose. Placebo group levels remained unchanged.

Conclusions: When administered daily for 29 days in patients with knee and/or hip OA, GLPG1972 at oral doses of 100, 200 and 300 mg q.d. was generally wel tolerated and safe. Serum ARGS levels, as a marker for target engagement and potential proxy of cartilage degradation, showed a dose-dependent decrease over time up to $53 \%$ below baseline in the $300 \mathrm{mg}$ group. These PK-PD findings are consistent with what we observed in a previous study in healthy subjects ${ }^{2}$ and reinforce the rationale for developing GLPG1972 as a DMOAD.

\section{REFERENCES:}

[1] Hunter DJ, et al. Curr. Opin. Rheumatol. 2009;21:110-117.

[2] van der Aar E, et al. Arthritis Rheumatol. 2017;69(suppl 10).

[3] Larsson, et al. Osteoarthritis Cartilage 2014;22(2):242-9. 
Disclosure of Interest: H. Deckx Employee of: Galapagos NV, Belgium, S Hatch Consultant for: Galapagos NV, Belgium, M. Robberechts Employee of: Galapagos NV, Belgium, S. Dupont Employee of: Galapagos SASU, France, J. Desrivot Employee of: has been employee of Galapagos SASU, France, H. Coleman Paid instructor for: Covance has been contracted by Galapagos NV to conduct the study, S. Larsson: None declared, A. Struglics: None declared, E. van der Aar Employee of: Galapagos NV, Belgium, A. Fiew Employee of: Galapagos NV, Belgium

DOI: 10.1136/annrheumdis-2018-eular.3101

\section{FRI0540 IDENTIFICATION OF BIOMARKERS OF OA ASSOCIATED TO DEFECTIVE AUTOPHAGY}

1. Lorenzo-Gomez ${ }^{1}$, N. Oreiro ${ }^{2}$, J.A. Pinto-Tasende ${ }^{2}$, F.J. Blanco ${ }^{1,2}$, B. Carames ${ }^{1}$. ${ }^{1}$ Rheumatology Division. Cartilage Biology Group, The Institute of Biomedical Research of A Coruña (INIBIC); ${ }^{2}$ Clinical Rheumatology Division, Complejo Hospitalario Universitario A Coruña (CHUAC), A coruña, Spain

Background: In osteoarthritis (OA), defects in cellular homeostasis, and in particular in autophagy, are evident and precede joint damage. In this sense, we have shown that there is a defect in autophagy in OA human chondrocytes and cartilage, and pharmacological activation of autophagy protects against joint damage. These data suggest that joint damage could be due, at least in part, to a failure of autophagy, by inducing an abnormal accumulation of cellular products related to disease.

Objectives: These observations represent a unique opportunity to identify and validate potential biomarkers associated with autophagy defects that could facilitate the development of therapeutic strategies to prevent OA progression.

Methods: A comparative analysis of 86 autophagy genes was performed in blood from non-OA and knee OA patients. Non-OA patients (Age: $61,17 \pm 1,370$ years; BMI: 25,76 $\pm 0,69$; Sex: Females; $n=12$ ) and Knee OA patients (Age: $65,75 \pm 1,528$ years; BMI: $30,25 \pm 0,88$; Sex: Females; $n=12$, OA grade III-IV) were profiled using a human autophagy PCR array (PrimePCR autophagy human panel, BioRad) and analysed using the PrimePCR analysis software, Biorad. In addition, we performed a quantitative proteomic analysis of defective autophagy by genetic deletion of Atg5 in human OA chondrocytes by using ITRAQ (isobaric tags for relative and absolute quantitation) labelling coupled with on-line 2D LC/MS/MS. Protein identification and quantification were performed using Protein Pilot Software 4.0. Each MS/MS spectrum was searched in the Uniprot/Swissprot database for Homo sapiens.

Results: 16 autophay-related genes were significantly down-regulated in blood from knee OA patients compared to non-OA patients. No significant up-regulation was observed in blood from Knee OA patients, however a trend-toward up-regulation was detected in several genes involved in the mTOR signalling pathway. Importanly, 5 key autophagy-related genes, such as, ATG16L2, ATG12, ATG7, ATG4B and MAP1LC3B involved in initiating autophagy, phagophore extension and autophagosome formation were significant downregulated in knee OA patients compared to non-OA patients $(p<0.05)$. Interestingly, HSP90AA1 and HSPA8, a chaperone-mediated autophagy genes involved in stress response and protein folding, were significant downregulated $(p<0.001)$ in blood from knee OA patients. In addition, several regulators of autophagy and apoptosis, such as BNIP3, BCL-2 and BCL2L1 were a significantly downregulated in OA patients $(p<0.01)$. Total proteome screening in human OA chondrocytes with defective autophagy, showed a significant reduction of Heat shock protein HSP90-alpha (HSP90A1) $(p<0.05)$, suggesting that reduced autophagy is associated to OA pathology and could be a potential biomarker for $O A$ progression and development.

Conclusions: This approach represents an unique opportunity to identify and validate early-stage biomarkers associated with defective autophagy that could facilitate the development of therapeutic strategies to prevent joint damage.

Disclosure of Interest: None declared

DOI: 10.1136/annrheumdis-2018-eular.5527

\section{FRI0541 INCREASING A PERSON'S OWN PHYSICAL ACTIVITY AND STRENGTH CAN MINIMISE CARTILAGE VOLUME LOSS IN OLDER-ADULTS: A BETWEEN- AND WITHIN- PERSON ANALYSIS ON A POPULATION-BASED PROSPECTIVE COHORT}

I.P. Munugoda ${ }^{1}$, S. Balogun ${ }^{1}$, K. Wills ${ }^{1}$, F. Cicuttini ${ }^{2}$, G. Jones ${ }^{1}$, M.L. Callisaya ${ }^{1}$, D. Aitken ${ }^{1} .{ }^{1}$ Menzies Institute for Medical Research, University of Tasmania, Hobart, Tasmania; ${ }^{2}$ Department of Epidemiology and Preventive Medicine, Monash University Medical School, Melbourne, Victoria, Australia

Background: The relationship between physical activity (PA) and osteoarthritis (OA) has been controversial, with some studies showing a detrimental effect and others showing either no effect or a beneficial effect. Traditionally, analysis has focused on examining the effect PA and/or strength have on OA between individuals (between-person comparison). Yet, how the variability in PA and strength over time within the same individual (within-person comparison) is associated with $O A$ is not well recognised. Statistical methods, such as multilevel models that properly capture the within-person processes can be used to tell us whether changes within an individual over time relate to changes in OA outcomes in that same individual.

Objectives: This study aimed to investigate the associations of between-person and within-person variability in PA and leg strength with knee cartilage volume loss over 10.7 years in older adults.

Methods: 479 community-dwelling older-adults (50\% female, mean age $61 \pm 6$ years, range $50-80$ years) were studied at baseline, $2.7,5.1$, and 10.7 years. PA (measured objectively as steps/day) and leg strength (measured objectively in $\mathrm{kg}$ ) were assessed at all four time-points. Knee cartilage volume was measured using MRI at baseline and 10.7 years. Linear mixed-effect regression models were used to estimate the association of between-person and within-person variability in PA and leg strength with cartilage volume loss over 10.7 years. Models were adjusted for age, sex, body mass index and history of knee injury or surgery.

Results: Mean cartilage volume loss over 10.7 years was $465 \pm 231 \mathrm{~mm}^{3}$. No between-person associations existed between PA and cartilage volume loss (Beta: 18.8 per 1000 steps/day, 95\% Cl -6.1, 43.7). However, within-person variability in PA was protectively associated with changes in cartilage volume, such that having higher PA compared to an individual's average level of PA minimised their cartilage volume loss over time (Beta: 32.8 per 1000 steps/day, 95\% Cl 20.8 44.6). Between-person effects showed that participants with greater leg strength lost less cartilage volume over time (Beta: 5.4 per $1 \mathrm{~kg}, 95 \% \mathrm{Cl} 3.1,7.8)$. Withinperson variability in leg strength was also protectively associated with changes in cartilage volume, such that having higher leg strength compared to an individual's average strength minimised their cartilage volume loss over time (Beta: 3.3 per $1 \mathrm{~kg}, 95 \% \mathrm{Cl} 2.1,4.5)$

Conclusions: Our unique analysis method adds a new perspective to the PA and OA debate. The implication of these findings demonstrate that individuals can minimise cartilage volume loss by increasing their own PA and strength, which supports the clinical recommendations of promoting PA and strength to prevent and treat $O A$

Disclosure of Interest: None declared

DOI: 10.1136/annrheumdis-2018-eular.3204

\section{FRI0542 POTENTIAL NOCICEPTIVE PAIN RELIEF OF INTRA- ARTICULAR SALINE CONTROL IN CLINICAL TRIALS OF KNEE OSTEOARTHRITIS: A SYSTEMATIC REVIEW AND META-ANALYSIS OF RANDOMISED TRIALS}

I. Simsek, T. Phalen, A. Bedenbaugh, J. Tambiah. Samumed, LLC, San Diego, USA

Background: Hyaluronic acid, corticosteroids and platelet-rich plasma (PRP) are widely used intra-articular $(\mathrm{IA})$ therapies for the management of mild to moderate knee osteoarthritis (OA). Many trials evaluating the efficacy of IA-administered therapies commonly use IA saline injections as a placebo comparator arm. A previously published systematic review Altman et al, 2016 showed significant reduc tions in pain relief with IA saline in both the short- (3 months) and long-term (6-12 months).

Objectives: The aim of this updated systematic review and meta-analysis was to assess the clinical benefit and harm associated with use of IA saline in trials of IA therapies for patients with painful knee OA

Methods: We searched MEDLINE and Embase databases for randomised controlled trials (RCTs) published up to and including October 12th, 2017. Two reviewers independently assessed the eligibility of potential reports and the risk of bias of included trials. We analysed short ( $\leq 3$ months) and long-term (6-12 months) pain reduction from baseline of the saline arm of included trials using standardised mean differences (SMDs; estimated assuming a null-effect in a comparator group) that were weighted and pooled using a random-effects model. Pain scores were transformed to a 100-point scale when necessary. We summarised and presented treatment-related adverse events (AEs) descriptively.

Results: We included 46 RCTs, of which 44 provided sufficient data to be included in the meta-analysis for benefit. IA saline significantly improved shortterm knee pain from baseline vs. a null effect for a comparator group across 36 studies involving 1908 patients (SMD $-0.85,95 \% \mathrm{Cl}-1.05$ to $-0.66 ; \mathrm{I}^{2}=87 \%$ ). There was also significant reduction in long-term knee pain following IA injection with saline across 25 studies involving 1758 patients (SMD $-0.78,95 \% \mathrm{Cl}-1.02$ to -0.55$)$ with a substantial degree of heterogeneity $\left(\mathrm{I}^{2}=90 \%\right)$. Thirty-three of the included trials reported on adverse events, none of which found any serious treatment-related AEs following IA injection with saline.

Conclusions: The pain relief observed with IA saline should prompt one to consider the added effectiveness of current IA treatments that use saline comparators in clinical studies, and challenges of classifying IA saline injection a "placebo." 